\title{
Sur les isomorphismes d'algèbres de restriction.
}

par

NOËL LEBLANC (Saint-Denis)

Résumé. Le but de cet article est de déterminer des conditions pour que deux ensembles totalement discontinus $E$ et $F$ puissent donner naissance à des algèbres de restriction $A(E)$ et $A(F)$ isomorphes. On étudie en particulier des conditions en rapport avec la multiplicité de $E$ et $F$.

Soit $E$, l'ensemble parfait symétrique construit à l'aide des rapports de dissection $\left\{\xi_{k}\right\}$ : on note $E_{0}=[0,1]$, puis $E_{1}=\left[0, \xi_{1}\right] \cup\left[1-\xi_{1}, 1\right]$, et on renouvelle l'opération précédente sur chacun des intervalles constituant $E_{1}$, pour définir $E_{2}$ avec le rapport $\xi_{2}, \ldots$ et ainsi de suite. Alors, si $E=\bigcap_{n \geqslant 0} E_{n}$,

$$
E=\left\{x ; x=\sum_{k \geqslant 1} \varepsilon_{k} \xi_{1} \ldots \xi_{k-1}\left(1-\xi_{k}\right), \varepsilon_{k} \epsilon\{0,1\}\right\} .
$$

Si $A(\boldsymbol{R})$ est l'algèbre des transformées de Fourier des fonctions de $L^{1}(\boldsymbol{R})$, si $I(E)$ est l'idéal des fonctions de $A(\boldsymbol{R})$ nulles sur $E$, on peut envisager l'algèbre quotient $A(E)=A(\boldsymbol{R}) / I(E)$, et il est naturel de se demander si cette algèbre peut être isomorphe à une algèbre $A(F)$, où $F$ est un ensemble fermé quelconque. Certains isomorphismes ont été mis en évidence (cf. [1], ch. IX), lorsque $E$ et $F$ sont des ensembles parfaits. symétriques dont les rapports de dissection tendent vers zéro, puis Y. Meyer a montré les limites de tels résultats [2].

Nous montrerons tout d'abord, dans une première partie, que, si $E$ satisfait une certaine condition de multiplicité, les homomorphismes de $A(F)$ dans $A(E)$ sont de la forme

$$
\mathfrak{S}: \Phi \rightarrow \Phi \circ f, \quad f(x)=\sum f_{k}(x),
$$

où la some est finie, et où, si l'on pose

$$
r_{j}=\xi_{1} \ldots \xi_{j-1}\left(1-\xi_{j}\right),
$$

$f_{k}(x)$ satisfait à la relation

$$
f_{k}\left(\sum_{j \geqslant 1} \varepsilon_{j} r_{j}\right)=\varepsilon_{j_{1}} \ldots \varepsilon_{j_{k}} f_{k}\left(r_{j_{1}}+\ldots+r_{j_{k}}\right),
$$


où la somme du second membre est étendue à tous les systèmes de $k$-indices possibles.

Pour préciser ce résultat, nous étudierons dans la seconde partie le cas où

$$
\lim _{k \rightarrow \infty} \xi_{k}=\frac{1}{2}
$$

Nous montrerons alors que les isomorphismes de $A(F)$ dans $A(E)$ sont définis par des fonctions linéaires au voisinage de tout point de $E$.

Dans la troisième partie, nous étudierons le cas où $f$ est une bijection entre deux ensembles parfaits symétriques $E$ et $F$, et nous donnerons une réponse négative à la question: si $E$ et $F$ sont deux ensembles parfaits symétriques tels que $E$ est de multiplicité et $F$ d'unicité, $A(E)$ et $A\left(F^{\prime}\right)$ peuvent-elles être isomorphes? Nous montrerons en fait ce résultat en utilisant des notions d'unicité et de multiplicité très particulières, mais l'étude de ces conditions montrera que, dans le cas particulier des ensembles parfaits symétriques définis par un rapport de dissection constant, les notions d'unicité et de multiplicité étudiées ici coincident avec les notions classiques.

\section{PREMIERE PARTIE}

Rappellons tout d'abord que tout homomorphisme de $A(F)$ dans $A(E)$ est de la forme

$$
\Phi \rightarrow \Phi \circ f
$$

et que le théorème du graphe fermé montre qu'une telle transformation définit effectivement un homomorphisme si et seulement si

$$
\|\Phi \circ f\|_{A(E)} \leqslant C\|\Phi\|_{A(F)},
$$

où $C$ est une constante indépendante de la fonction $\Phi$.

Nous utiliserons ici une méthode voisine de celle de Beurling et Helson [3] pour conclure tout d'abord lorsque $E$ est parfait symétrique:

THÉORÈmE I,1. Si $E$ porte une mesure positive $\lambda$ satisfaisant

les fonctions telles que

$$
\lim _{|t| \rightarrow \infty} \hat{\lambda}(t)=0
$$

$$
\left\|e^{n i f}\right\|_{A(E)} \leqslant C
$$

pour une certaine constante $C$ indépendante de $n$, satisfont une relation

$$
\sum(-1)^{J} f\left(\sum_{j=1}^{N} \varepsilon_{j} x_{j}\right)=0, \quad J=\sum_{j=1}^{N} \varepsilon_{j},
$$

où la première somme est étendue à tous les systèmes de valeurs de $\varepsilon_{\mathrm{j}}$ possible, avec $\varepsilon_{j} \in\{0,1\}$, et où $N$ est une constante dépendant de $f$.

Démonstration. Soit $v$, la mesure de masse $\frac{1}{4}$ en 0 , $\frac{1}{4}$ en $a_{1}, \frac{1}{4}$ en $a_{2}$ $-\frac{1}{4}$ en $a_{1}+a_{2}$. Un calcul élémentaire montre que

$$
\int d|\nu|=\|v\|_{M}=1, \quad \sup _{t}|\hat{\nu}(t)|=\|v\|_{P M} \leqslant \frac{\sqrt{2}}{2} .
$$

Nous envisageons ici la mesure

$$
\omega=v_{1} * v_{2} * \ldots * v_{2 K}
$$

portée par $4^{2 K}$ points distincts de $E$, où les $v_{j}$ sont construites comme précédemment. Alors,

$$
\|\omega\|_{M}=1, \quad\|\omega\|_{P M} \leqslant 2^{-K},
$$

et, si $f$ est une fonction définie sur $E$ telle que

$$
\left\|e^{n i f}\right\|_{A(E)}<2^{K-1}
$$

on a nécessairement

$$
\int e^{n i f} d \omega<\frac{1}{2}
$$

et le théorème de Kronecker entraîne l'existence d'une relation

$$
\begin{aligned}
\sum_{i=1}^{42 K} A_{i} f\left(t_{i}\right) & =0, \quad A_{i} \epsilon\{-2,-1,0,1,2\}, \\
t_{i} & =\sum \varepsilon_{j} x_{j}, \quad \varepsilon_{j} \in\{0,1\},
\end{aligned}
$$

où les $x_{j}$ sont les $4 K$ points de $E$ qui interviennent dans la construction de la mesure $\omega$. On constate alors que le nombre de relations possibles de ce type est fini, et l'on définit

$$
g(x)=\sum_{i=1}^{4^{2 K}} A_{i} f\left(t_{i}\right),
$$

où les $A_{i}$ et les $t_{i}$ sont comme ci-dessus. Le domaine de définition de.g est le sous ensemble de $E^{4 K}$ formé des suites de $4 K$ éléments de $E$ tels que

$$
\sum_{j=1}^{4 K} \varepsilon_{j} x_{j} \in E, \quad \forall \varepsilon_{j} \in\{0,1\} .
$$

Soit $E^{\prime}$, ce sous ensemble; si $g$ ne s'annule sur aucun pavé de $E^{\prime}$,

$$
\overline{\left\{x \in E^{\prime} ; g(x) \neq 0\right\}}=E^{\prime},
$$


et, commè le théorème de Kronecker implique l'existence en tout point de $E^{\prime}$, d'une relation qui a lieu sur un fermé de $E^{\prime}$, il existe sur

$$
\left\{x \in E^{\prime} ; g(x)=0\right\}
$$

une autre relation. Comme le nombre des relations possibles est fini, on pourra trouver une relation ayant lieu sur un pavé de $E^{\prime}$. On peut donc supposer que $g$ s'annule sur un paré de $E^{\prime}$, si les coefficients $A_{i}$ ont été choisis de façon convenable. En répétant le raisonnement précédent sur le complémentaire de ce pavé, on pouri’a même supposer que $g$ s'annule sur une suite infinie de pavés de $E^{\prime}$ ayant 0 comme point d'accumulation.

Supposons alors que $g$ n'est identiquement nulle dans aucun voisinage de 0 . Il existe deux suites infinies de parés ayant zéro comme point d'accumulation et telles que

$$
\begin{array}{ll}
\left\{P_{n}\right\} ; & g(x)=0 \operatorname{sur} P_{n}, \\
\left\{Q_{n}\right\} ; & g(x) \neq 0 \operatorname{sur} Q_{n} .
\end{array}
$$

Comme il existe une mesure positive portée par $E$ dont la transformée de Fourier tend vers 0 à l'infini, on peut trouver, quitte à extraire des sous-suites de $\left\{P_{n}\right\}$ et $\left\{Q_{n}\right\}$, des mesures unitaires positives $\mu_{n}^{+}$et $\mu_{n}^{-}$respectivement portées par $P_{n}$ et $Q_{n}$, telles que

$$
\sigma_{N}=\sum_{n=1}^{N} \mu_{n}^{+}-\mu_{n}^{-}
$$

satisfait l'inégalité

$$
\left\|\sigma_{N}\right\|_{P M\left(E^{\prime}\right)} \leqslant 4
$$

On obtient alors, pour $p$ assez grand dépendant de $N$,

$$
\left\|\cos ^{p} g\right\|_{A\left(E^{\prime}\right)} \geqslant \frac{1}{4} \int_{E^{\prime}} \cos ^{p} g d \sigma_{N} \geqslant \frac{N}{8},
$$

et donc, si l'on a choisi $N$ assez grand,

$$
\left\|e^{p i g}\right\|_{\mathcal{A}\left(E^{\prime}\right)} \geqslant\left(2^{\mathcal{K}}\right)^{\left(4^{2 K}\right)}
$$

il existe donc une suite $\left\{\varepsilon_{j}\right\}$ fixée telle que

$$
\left\|e^{p i f}\right\|_{A(E)} \geqslant\left\|e^{p i f\left(\Sigma e_{j} x_{j}\right)}\right\|_{A\left(E^{\prime}\right)} \geqslant 2^{K}
$$

et nous obtenons donc une contradiction avec 1'hypothèse, ce qui nous montre que $g$ est identiquement nulle dans un voisinage de zéro de $E^{\prime}$.

Quitte à choisir une autre relation, nous pourons en outre supposer que $g(x)=0$ est la relation valable sur un voisinage de zéro, et telle que $A_{i}=0$ dès que $J=\sum \varepsilon_{j}$ dépasse l'entier $M$ choisi aussi petit que possible (ou, s'il en existe plusieurs correspondant au même $M$, l'une des relations contenant le plus petit nombre de $A_{i}$ non nuls avec $J=M$ ). Si pour un $j$ fixé, nous choisissons $x_{j}=0$, nous obtenons une nouvelle relation; d'après le choix que nous avons fait, la nouvelle relation. est nécessairement triviale. Ceci nous montre que la relation $g(x)=0$ est

$$
\sum(-1)^{J} f\left(\sum_{j=1}^{M} \varepsilon_{j} x_{j}\right)=0, \quad J=\sum_{j=1}^{M} \varepsilon_{j}
$$

qui entraîne la même relation dans laquelle on remplace $I I$ par $4 K$, et cette dernière relation a lieu dès que $\sum \varepsilon_{j} x_{j}$ est assez petit. La même démonstration permettrait d'ailleurs d'établir, pour $x_{0} \in E$ fixé,

$$
\sum(-1)^{J} f\left(x_{0}+\sum_{j=1}^{4 K} \varepsilon_{j} x_{j}\right)=0, \quad J=\sum_{j=1}^{4 K} \varepsilon_{j},
$$

et cette dernière relation a lieu dès que $x_{0}+\sum \varepsilon_{j} x_{j}$ appartient à un segment de la forme $\left[y_{0}, y_{0}+\xi_{1} \ldots \xi_{k_{0}}\right]$, où $y_{0}$ est combinaison linéaire des $\xi_{1} \ldots$ $\ldots \xi_{i-1}\left(1-\xi_{i}\right), i \leqslant k_{0}$. On vérifie alors que si $x_{0}^{\prime}$ appartient au segment $\left[y_{0}, y_{0}+\xi_{1} \ldots \xi_{k_{0}}\right]$, la relation précedente a encore lieu lorsqu'on remplace $x_{0}$ par $x_{0}^{\prime}$, et qu'on impose à $x_{0}^{\prime}+\sum \varepsilon_{j} x_{j}$ d'appartenir encore au segment.

On associe done à chaque $x$ de $E$ le segment construit comme ici, et on on obtient ainsi un recouvrement de $E$ d'où l'on extrait un recouvrement fini. En choisissant le plus grand indice $k$ correspondant au $k_{0}$ précédent, et en subdivisant certain des segments obtenus, la relation a encors lieu si $x_{0}+\sum \varepsilon_{j} x_{j}$ appartient à l'un quelconque des segments. $\left[y, y+\xi_{1} \ldots\right.$

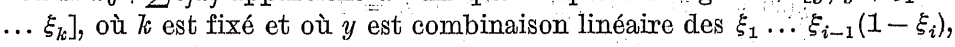
$i \leqslant k$. On peut alors assurer

$$
\sum(-1)^{J} f\left(\sum_{j=1}^{4 K+k} \varepsilon_{j} x_{j}\right)=0, \quad J=\sum_{j=1}^{4 K+k} \varepsilon_{j},
$$

puisque cette somme se décompose en $2^{k}$ sommes partielles qui sont toutes nulles d'après le résultat précédent; nous obtenons donc la relation annoncée arec $N=4 K+k$.

Nous pouvons alors chercher à résoudre cette relation:

THÉORÈME $\mathrm{I}, 2$. Si $E$ porte ine mesure positive $\lambda$ satisfaisant

$$
\lim _{t \rightarrow \infty} \hat{\lambda}(t)=0,
$$

les fonctions telles que

$$
\left\|e^{n i f}\right\|_{A(E)} \leqslant C
$$


pour une certaine constante $C$ indépendante de $n$, sont de la forme

$$
f(x)=\sum f_{k}(x)
$$

où la somme est finie, et où, si l'on pose

$$
r_{j}=\xi_{1} \ldots \xi_{j-1}\left(1-\xi_{j}\right)
$$

\section{$f_{k}$ satisfait la relation}

$$
f_{k}\left(\sum_{j \geqslant 1} \varepsilon_{j} r_{j}\right)=\sum \varepsilon_{j_{1}} \ldots \varepsilon_{j_{k}} f_{k}\left(r_{j_{1}}+\ldots+r_{j_{k}}\right),
$$

où la somme du second membre est étendue à tous les systèmes de $k$ indices possibles.

Démonstration. Pour résoudre la relation que nous avons trouvée dans l'étude précédente, nous remarquons qu'il nous suffit d'obtenir une fonction satisfaisant la même relation, et prenant la valeur de $f(x)$ aux points $x$ de $E$ de la forme

Nous posons alors

$$
x=\sum \varepsilon_{i} r_{j}, \quad \sum \varepsilon_{j}<N .
$$

$$
f_{0}(x)=f(0),
$$

et nous définissons par récurrence, pour $x=\sum \varepsilon_{j} r_{j}$, et $k<N$,

$$
\begin{aligned}
& f_{k}(x)=0 \quad \text { si } \sum \varepsilon_{j}<k, \\
& f_{k}(x)=f(x)-\sum_{j<k} f_{j}(x) \quad \text { si } \sum \varepsilon_{j}=k, \\
& f_{k}(x)=\sum \varepsilon_{j_{1}} \ldots \varepsilon_{j_{k}} f_{k}\left(r_{j_{1}}+\ldots+r_{j_{k}}\right) \quad \text { si } \sum \varepsilon_{j}>k .
\end{aligned}
$$

On vérifie alors que, pour tout point $x$ tel que $\sum \varepsilon_{j}<N$,

$$
f(x)=\sum f_{k}(x) \text {. }
$$

Il reste donc à montrer que $f_{k}(x)$ est solution de

$$
\sum(-1)^{J} f\left(\sum_{j=1}^{N} \varepsilon_{j} x_{j}\right)=0, \quad J=\sum_{j=1}^{N} \varepsilon_{j}
$$

ce qui entraîne que $f(x)$ en est aussi solution, puisque toute combinaison linéaire de solutions est solution. Or $f_{k}(x)$ s'écrit comme une combinaison linéaire de termes de la forme

$$
f_{k}\left(r_{i_{1}}+\ldots+r_{i_{k}}\right)
$$

et le coefficient de ce terme dans

$$
\sum(-1)^{J} f_{k}\left(\sum_{j=1}^{N} \varepsilon_{j} x_{j}\right)
$$

est, si les atomes $r_{i_{1}}, \ldots, r_{i_{k}}$ proviennent de $x_{j_{1}}, \ldots, x_{j_{h}}$,

$$
\sum_{J=h}^{N}(-1)^{J}\left(\begin{array}{l}
N-h \\
J-h
\end{array}\right)=0
$$

ce qui achève la démonstration.

\section{DEUXIÉME PARTIE}

Nous supposons dans cette partie que la suite des rapports de dissection définissant $E$ a pour limite $\frac{1}{2}$, et nous démontrons tout d'abord que les résultats de la première partie s'appliquent à cette situation:

LEMNE II, 1. Si la suite des rapports de dissection $\left\{\xi_{k}\right\}$ définissant $E$ a pour limite $\frac{1}{2}$, la mesure unitaire $\lambda$ équirépartie sur $E$ satisfait

$$
\lim _{|t| \rightarrow \infty} \hat{\lambda}(t)=0 \text {. }
$$

Démonstration.

$$
\begin{aligned}
|\hat{\lambda}(t)| & =\prod_{k \geqslant 1}\left|\cos \xi_{1} \ldots \xi_{k-1}\left(1-\xi_{k}\right) \frac{t}{2}\right| \\
& \leqslant \prod_{q+r \geqslant k>q}\left|\cos 2^{q-k} \xi_{1} \ldots \xi_{q}\left(1-\varepsilon_{k}\right) \frac{t}{2}\right|,
\end{aligned}
$$

et nous pouvons majorer $\varepsilon_{k}$ à l'aide de la formule des accroissements finis pour les fonctions de plusieurs variables

$$
\left|\varepsilon_{k}\right| \leqslant \sum_{k \geqslant j>q}\left(1-2 \xi_{j}\right) \leqslant(k-q) \sup _{k \geqslant q}\left(1-2 \xi_{k}\right)
$$

puis $\hat{\lambda}(t)$, par la même formule

$$
\begin{gathered}
|\hat{\lambda}(t)| \leqslant \prod_{q+r \geqslant k>q}\left|\cos 2^{q-k} \xi_{1} \ldots \xi_{q} \frac{t}{2}\right|+\sum_{q+r \geqslant k>q} 2^{q-k} \xi_{1} \ldots \xi_{q}\left|\varepsilon_{k} \frac{t}{2}\right| ; \\
|\hat{\lambda}(t)| \leqslant\left|\frac{2^{-r} \sin \xi_{1} \ldots \xi_{q} \frac{t}{2}}{\sin 2^{-r} \xi_{1} \ldots \xi_{q} \frac{t}{2}}\right|+\xi_{1} \ldots \xi_{q} \sup _{k \geqslant q}\left(1-2 \xi_{k}\right)|t| .
\end{gathered}
$$


Pour $r$ fixé, nous déterminons tout d'abord $q_{0}$ tel que

$$
\sup _{k \geqslant q_{0}}\left(1-2 \xi_{k}\right) \leqslant 2^{-2 r}
$$

puis, pour $|t| \geqslant 2^{r} \xi_{1}^{-1} \ldots \xi_{q_{0}}^{-1}$, nous choisissons $q \geqslant q_{0}$ tel que

$$
1 \leqslant 2^{-r} \xi_{1} \ldots \xi_{q}|t| \leqslant 2 \text {. }
$$

Nous pourons alors déduire de (1)

$$
|\hat{\lambda}(t)| \leqslant 2^{-r+2}
$$

ce qui est le résultat puisque $r$ est arbitraire.

Nous définissons alors les mesures suivantes, où $n$ est un entier fixé, supérieur à 10:

$\mu_{p}$, qui affecte de la masse 1 chacun des points de la forme

$\sum_{k=1}^{n} \varepsilon_{k} \xi_{1} \ldots \xi_{p+k-1}\left(1-\xi_{p+k}\right)+\frac{1}{2} \xi_{1} \ldots \xi_{p+n}, \quad \varepsilon_{k} \epsilon\{0,1\}, \varepsilon_{2}=\ldots=\varepsilon_{10}=1-\varepsilon_{1} ;$

$v_{p}$, translatée de la mesure unitaire équirépartie sur $E \cap\left[0, \xi_{1} \ldots \xi_{p+n}\right]$ par la translation qui transforme cet ensemble en un ensemble symétrique par rapport à l'origine;

$\sigma_{p}$, le produit de convolution

$$
\frac{\mu_{p}}{x-\frac{1}{2} \xi_{1} \ldots \xi_{p}} * v_{p}
$$

$\sigma_{p}$ est alors une mesure portée par $E$ telle que

LEMINEE II, 2. $S i$

$$
\sup _{q>p}\left(1-2 \xi_{q}\right) \leqslant n^{-2} 4^{-n}
$$

alors

$$
\left\|\sigma_{p}\right\|_{P M}=\sup _{t}\left|\hat{\sigma}_{p}(t)\right| \leqslant \frac{7}{\xi_{1} \ldots \xi_{p+n}} .
$$

Démonstration. Comme

$$
\left|\hat{v}_{p}(t)\right|=\prod_{k>p+n}\left|\cos \xi_{1} \ldots \xi_{k-1}\left(1-\xi_{k}\right) \frac{t}{2}\right|,
$$

(1) est vérifié aree $\nu_{p}$ au lieu de $\lambda$, dès que $q \geqslant p+n$, et un calcul identique à celui du lemme 1 donne alors

$$
|t| \geqslant \frac{n}{\xi_{1} \ldots \xi_{p+n}} \quad \text { entraîne }\left|\hat{v}_{p}(t)\right| \leqslant \frac{4}{n}
$$

ce qui amélioire la majoration triviale

$$
\left|\hat{v}_{p}(t)\right| \leqslant\left\|\nu_{p}\right\|_{P M} \leqslant\left\|\nu_{p}\right\|_{M}=1 .
$$

Nous pouvons par ailleurs améliorer aussi la majoration

(4) $\quad\left|\left(\frac{\mu_{p}}{x-\frac{1}{2} \xi_{1} \ldots \xi_{n}}\right) \wedge(t)\right| \leqslant\left\|\frac{\mu_{p}}{x-\frac{1}{2} \xi_{1} \ldots \xi_{p}}\right\|_{M I} \leqslant \frac{2 n \log 2}{\xi_{1} \ldots \xi_{p+n}}:$

soit $\varrho_{p}$, la mesure qui se déduit de $\mu_{p}$ par la translation qui amène $\frac{1}{2} \xi_{1} \ldots$ $\ldots \xi_{p}$ à l'origine:

$$
\left|\left(\frac{\mu_{p}}{x-\frac{1}{2} \xi_{1} \ldots \xi_{p}}\right)^{\wedge}(t)\right|=\left|\int_{0}^{t} \hat{\varrho}_{p}(s) d s\right|,
$$

et, si nous définissons $\varepsilon_{k}$ comme dans le lemme 1 ,

$$
\int_{0}^{t} \hat{\varrho}_{p}(s) d s=\int_{0}^{t} 2^{n-9} \prod_{n \geqslant k \geqslant 10} \cos 2^{-k} \xi_{1} \ldots \xi_{p}\left(1-\varepsilon_{k}\right) \frac{s}{2} d s,
$$

ce qui nous permet d'obtenir, par un calcul analogue à celui qui nous a permis d'établir (1)

$$
\left|\int_{0}^{t} \hat{\varrho}_{p}(s) d s\right| \leqslant\left|\int_{0}^{t} \frac{\sin 2^{-10} \xi_{1} \ldots \xi_{p} s}{\sin 2^{-n-1} \xi_{1} \ldots \xi_{p} s} d s\right|+\frac{3}{8} 2^{-n-1} n^{-2} \xi_{1} \ldots \xi_{p} t^{2} .
$$

Le lemme d'Abel nous permet de majorer l'intégrale:

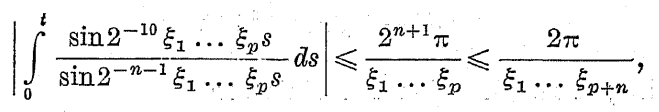

tandis que l'hypothèse du lemme implique en outre

On obtient alors

$$
\frac{2^{-n}}{\xi_{p+1} \ldots \xi_{p+n}} \leqslant \frac{9}{8}
$$

$$
\left|\int_{0}^{t} \hat{\varrho_{p}}(s) d s\right| \leqslant \frac{2 \pi}{\xi_{1} \ldots \xi_{p+n}}+\frac{1}{4} n^{-2} \xi_{1} \ldots \xi_{p+n} t^{2}
$$

(5) $\quad|t| \leqslant \frac{n}{\xi_{1} \ldots \xi_{p+n}} \quad$ entraîne $\left|\left(\frac{\mu_{p}}{x-\frac{1}{2} \xi_{1} \ldots \xi_{p}}\right)^{\wedge}(t)\right| \leqslant \frac{7}{\xi_{1} \ldots \xi_{p+n}}$.

Le lemme découle alors immédiatement de (2), (3), (4) et (5). 
Remarque. Un calcul plus précis de (2) et (5) nous aurait permis, dans l'énoncé du lemme, de remplacer $n^{-2} 4^{-n}$ par $n^{-1} 2^{-n}$. Nous ne ferons pas ce calcul ici, car nous n'aurons pas besoin d'une telle précision.

Le lemme va nous permettre de préciser les théorèmes de la première partie, dans le cas particulier que nous envisageons ici; nous allons toutefois oublier tout d'abord ces résultats, et établir une proposition en utilisant seulement le lemme. Pour cela, nous rappelons que $E$ peut être considéré comme l'intersection d'une suite d'ensembles $E_{k}$, chaque $E_{k}$ étant constitué de $2^{k}$ intervalles de longueur $\xi_{1} \ldots \xi_{k}$. Si $I_{k}$ est l'un quelconque de ces intervalles, nous notons $I_{k, j}$ les intervalles, rangés dans l'ordre des $j$ croissants, qui constituent $I_{k} \cap E_{k+10}\left(1 \leqslant j \leqslant 2^{10}\right)$, puis

$$
F_{k, j}=\left[\inf f\left(I_{k, j} \cap E\right), \sup f\left(I_{k, j} \cap E\right)\right] \text {. }
$$

Proposition II,1. S'il existe une constante $C$ telle que

$$
\nabla \Phi \in A\left(F^{\prime}\right), \quad\|\Phi \circ f\|_{A(E)} \leqslant O\|\Phi\|_{A(F)},
$$

alors, quel que soit l'intervalle $I_{k}$ choisi à la $k^{i e ̀ m e}$ étape,

$$
\lim _{k \rightarrow \infty} \sup _{j} \frac{\operatorname{dist}\left(F_{k, j}, F_{k, j+1}\right)}{\operatorname{diam}\left(F_{k, j}\right)}=0
$$

et le résultat est conservé si $F_{k, j+1}$ remplace au dénominateur $F_{k, j}$.

Démonstration. Si cette inégalité n'avait pas lieu, il existerait une constante strictement positive $\eta$ telle que, quel que soit l'entier $n$, on pourrait trouver des indices $k$ et $j$ satisfaisant

$$
\begin{gathered}
\eta \operatorname{dist}\left(F_{k, j}, F_{k, j+1}\right) \geqslant \operatorname{diam}\left(F_{k, j}\right), \\
\sup _{q>k}\left(1-2 \xi_{q}\right) \leqslant n^{-2} 4^{-n} .
\end{gathered}
$$

Si nous envisageons la fonction $\Phi$ définie par

$$
\Phi(z)=\left\{\begin{array}{l}
1 \text { si } z \in F_{k, j}, \\
0 \text { si } \operatorname{dist}\left(z, F_{k, j}\right) \geqslant \eta^{-1} \operatorname{diam}\left(F_{k, j}\right), \\
\text { linéaire dans les intervalles, }
\end{array}\right.
$$

un calcul élémentaire nous donne

$$
\|\Phi\|_{A\left(F^{\prime}\right)} \leqslant\|\Phi\|_{A(\boldsymbol{R})} \leqslant 2+\log (\eta+1) .
$$

Quitte à envisager une mesure translatée de $\sigma_{k}$, pour laquelle le calcul du lemme serait encore valable, on peut alors supposer $j=2^{9}$, d'où

$$
\int_{E} \Phi \circ f d \sigma_{k}=\int_{1 \xi_{1} \ldots \xi_{k}}^{\xi_{1} \ldots \xi_{k}} \frac{d \mu_{k}}{x-\frac{1}{2} \xi_{1} \ldots \xi_{k}} \geqslant \frac{(n-10) \log 2}{\xi_{1} \ldots \xi_{k+n}}
$$

et nous obtenons, en tenant compte du lemme 2

$$
\|\Phi \circ f\|_{A(E)} \geqslant \frac{(n-10) \log 2}{7}
$$

(6) et (7) nous donnent alors une contradiction avec 1'hypothèse puisqup $\eta$ est fixé et que $n$ peut être choisi arbitrairement grand.

Let résultats que nous avons établis jusqu'ici s'appliquent à tous les homomorphismes de $A\left(F^{\prime}\right)$ dans $A(E)$. Pour obtenir des renseignements plus précis, nous allons introduire maintenant la notion d'isomorphisme. En fait, nous utiliserons seulement la propriété suivante: les isomorphismes de $A\left(F^{\prime}\right)$ dans $A(E)$ sont de la forme $\Phi \rightarrow \Phi \circ f$, où $f$ est une injection de $E$ sur $F$ telle que

$$
\mathbb{H} C, \nabla \Phi \epsilon A(F),\|\Phi \circ f\|_{A(E)} \leqslant C\|\Phi\|_{A(H)} .
$$

Propostition II,2. Si $f$ définit un isomorphisme de $A(F)$ dans $A(E)$,

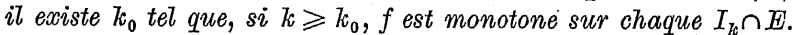

Démonstration. Nous pourrons conclure si nous savons montrer que, pour $k$ assez grand, les $F_{k, j}$ sont rangés dans l'ordre des $j$ croissants (ou décroissant). Nous écrirons

$$
F_{k, j}<F_{k, j^{\prime}} \quad \text { si } \sup \left(F_{k, j}\right)<\inf \left(F_{k, j^{\prime}}\right) .
$$

Si la proposition n'était pas réalisée, les $F_{k, j}$ seraient soit rangés en désordre, soit non totalement ordonnés. Dans le premier cas, on aurait par exemple

$$
F_{k, j}<F_{k, i}<F_{k, j+1}<F_{k, i+1},
$$

ce qui est impossible d'après la proposition 1 , si $k_{0}$ est assez grand pour que

$$
k \geqslant k_{0} \quad \text { entraîne } \sup _{j} \frac{\operatorname{dist}\left(F_{k, j}, F_{k, j+1}\right)}{\operatorname{diam}\left(F_{k, j}\right)}<1 .
$$

La proposition ne peut donc être en défaut que dans le cas où l'on peut trouver $i$ et $j$ distincts tels que $F_{k, i} \cap F_{k, j} \neq \varnothing$.

L'hypothèse (8) nous permet alors de trouver $k^{\prime}>k$ tel qu'il existe

$$
\begin{aligned}
& F_{k^{\prime}, i^{\prime}} \subset F_{k, i}, \\
& F_{k^{\prime}, j^{\prime}} \subset F_{k, j}, \\
& F_{k^{\prime}, i^{\prime}} \cap F_{k^{\prime}, j^{\prime}} \neq \varnothing,
\end{aligned}
$$

et de construire ainsi une suite de segments emboités ayant pour limite commune

avec

$$
f\left(x_{1}\right)=f\left(x_{2}\right)
$$

$$
x_{1} \in I_{k d} x_{2} \in I_{k, j}
$$


et donc $x_{1} \neq x_{2}$, ce qui est impossible puisque nous supposons ici que $f$ est une injection.

Corollarre. Si $f$ définit un isomorphisme de $A(F)$ dans $A(E)$, si $N$ est la constante qui intervient dans le théorème I, 1 , la fonction

$$
g(x)=\sum(-1)^{J}\left[f\left(\sum_{j=1}^{N-2} \varepsilon_{j} r_{j}+x\right)-f\left(\sum_{j=1}^{N-2} \varepsilon_{j} r_{j}\right)\right]
$$

est une function à variations bornées, définie pour

$$
x \in E^{\prime}=\left\{x \in E ; x<\inf _{j}\left\{r_{j}\right\}\right\}, \quad r_{j}=\xi_{1} \ldots \xi_{j-1}\left(1-\xi_{j}\right)
$$

telle que, si $F^{\prime}=g\left(E^{\prime}\right)$, il exrste une constante $C^{\prime}$,

et que

$$
\nabla \Phi \in A\left(F^{\prime}\right), \quad\|\Phi \circ g\|_{\Delta\left(E^{\prime}\right)} \leqslant C^{\prime}\|\Phi\|_{\mathcal{A}\left(F^{\prime}\right)},
$$

$$
g(x+y)=g(x)+g(y) \text {. }
$$

Si l'on note $u_{k}=g\left(r_{k}\right), 2^{k} u_{k}$ reste alors borné indépendamment de $k$.

Démonstration. D'après la proposition 2, $g$ est combinaison linéaire de fonctions à variations bornées, et est donc elle-même à variations bornées. Comme

$$
\left|2^{-N} 2^{k} u_{k}\right| \leqslant \operatorname{var}(g)
$$

$2^{k} u_{k}$ reste borné. Les autres résultats découlent immédiatement du théorème $I, 1$.

Proposition II,3. La suite $u_{k}=g\left(r_{k}\right)$ est telle qu'il existe $k_{1}$, avec l'une des propriétés suivantes, pour $k \geqslant k_{1}$

$$
\begin{gathered}
u_{k}=0 \\
\left|2 \frac{u_{k+1}}{u_{k}}-1\right| \leqslant \frac{1}{10} .
\end{gathered}
$$

Démonstration. La proposition 1 s'applique à la fonction $g$, et nous permet de déterminer $k_{1}$ tel que $k \geqslant k_{1}$ entraîne

$$
\left|u_{k}-u_{k+1}-\ldots-u_{k+9}\right| \leqslant 2 v_{k+9} \text {, }
$$

où $v_{l c}$ représente le reste de la série de terme général $\left|u_{l o}\right|$; en utilisant cette inégalité aux ordres $k$ et $k+1$, on obtient

$$
\left|u_{k}-2 u_{k+1}\right| \leqslant 4 v_{k+9} \text {. }
$$

Le corollaire de la proposition 2 nous permet en outre de déterminer, pour $\varepsilon$ fixé entre 0 et 1 , une suite infinie d'indices $m \geqslant k_{1}+10$, tels que

$$
\sup _{j=m} 2^{j}\left|u_{j}\right| \leqslant(1+\varepsilon) 2^{m}\left|u_{m}\right| \text {. }
$$

Ceci nous donne, en supposant $u_{m} \geqslant 0$,

$$
v_{m+8} \leqslant(1+\varepsilon) 2^{-8} u_{m}
$$

et, en reportant dans (9), avec $k=m-1$,

$$
\left|u_{m-1}-2 u_{m}\right| \leqslant(1+\varepsilon) 2^{-6} u_{m},
$$

ce qui entraine $u_{m-1} \geqslant 0$, et, si $u_{m}>0$,

$$
\frac{2}{2+(1+\varepsilon) 2^{-6}} \leqslant 2 \frac{u_{m}}{u_{m-1}} \leqslant \frac{2}{2-(1+\varepsilon) 2^{-6}} ;
$$

comme $\varepsilon$ est compris entre 0 et 1 ,

$$
\left|2 \frac{u_{m}}{u_{m-1}}-1\right| \leqslant(1+\varepsilon) 2^{-6}
$$

et nous pouvons remplacer (10) par

$$
\sup _{j \geqslant m} 2^{j}\left|u_{j}\right| \leqslant\left(1+2^{-6}+\frac{11}{10} \varepsilon\right) 2^{m-1} u_{m-1} .
$$

Nous pouvons alors, si $\varepsilon$ a été choisi assez petit (en fait $\varepsilon=\frac{1}{5}$ convient), renouveler le calcul précédent avec (9) et (11), et ainsi de suite, pour obtenir, si $m-10 \leqslant j \leqslant m$,

$$
\left|2 \frac{u_{j}}{u_{j-1}}-1\right| \leqslant \frac{1}{10} .
$$

Si nous supposons par récurrence que, si $m-10 \geqslant k \geqslant k_{1}$, si $k+2 \leqslant j$. $\leqslant m$,

$$
\left|2 \frac{u_{j}}{u_{j-1}}-1\right| \leqslant \frac{1}{10}
$$

(9) devient, compte tenu de ce dernier résultat et de (10)

$$
\left|u_{k}-2 u_{k+1}\right| \leqslant 4 v_{k+9} \leqslant 4 u_{k+1} \sum_{n \geqslant 8}\left(\frac{6}{10}\right)^{n} \leqslant \frac{u_{k+1}}{50},
$$

ce qui entraîne

$$
\left|2 \frac{u_{k+1}}{u_{k}}-1\right| \leqslant \frac{1}{10}
$$

et vérifie donc l'hypothèse de récurrence, et achève la démonstration puisque $m$ peut être choisi arbitrairement grand.

Remarque. Nous aurions pu obtenir une majoration plus précise, mais nous n'en aurons pas besoin dans la suite. D'ailleurs, pour démontrer 
à ce stade le meilleur résultat possible, c'est à dire

$$
\lim _{k \rightarrow \infty} \frac{u_{k+1}}{u_{k+}}=\frac{1}{2}
$$

(résultat que nous retrouverons par la suite), il aurait fallu démontrer le lemme 2 sous une forme plus précise, en faisant disparaitre la constante 10 qui intervient dans la définition de $\mu_{p}$.

COROLLATRE. En se limitant aux $k \geqslant k_{1}$, on peut poser

avec

$$
u_{k}=\zeta_{1} \ldots \zeta_{k-1}\left(1-\zeta_{k}\right)
$$

$$
\left|\zeta_{k}-\frac{1}{2}\right| \leqslant \frac{1}{20} .
$$

Démonstration. Il suffit de poser (si $u_{k}$ reste positif)

$$
v_{k}=\zeta_{1} \ldots \zeta_{k}
$$

(si $u_{k}$ reste négatif, on prendra pour $v_{k}$ l'opposé de cette valeur). On obtient alors

$$
u_{k}=v_{k-1}-v_{k}=\zeta_{1} \ldots \zeta_{k-1}\left(1-\zeta_{k}\right)
$$

Commé nous savons

$$
\begin{aligned}
& \frac{9}{20} \leqslant \frac{u_{k+1}}{u_{k}} \leqslant \frac{11}{20}, \\
& \frac{9}{11} \leqslant \frac{v_{k}}{u_{k}}=\frac{\zeta_{k}}{1-\zeta_{k}} \leqslant \frac{11}{9}
\end{aligned}
$$

et done $9 \leqslant 20 \zeta_{k} \leqslant 11$, ce qui est le résultat annoncé.

Proposimion II, 4. Si $u_{k}$ n'est pas identiquement nul, et est mis sous $l a$ forme

$$
u_{k}=\zeta_{1} \ldots \zeta_{k-1}\left(1-\zeta_{k}\right)
$$

il existe $k_{2}$ tel que $k \geqslant k_{2}$ entraîne $\zeta_{k}=\xi_{k}$.

Démonstration. Soit

$$
\Phi(z)=\exp \frac{i \frac{\pi}{2} z}{\zeta_{1} \ldots \zeta_{p}\left(\xi_{p+1}-\zeta_{p+1}\right)} .
$$

$\|\Phi\|_{A(F)} \leqslant 1$, et, si nous envisageons la mesure $\sigma_{p}$ qui intervient dans le lemme 2, les éléments du support de $\sigma_{p}$ qui satisfont $x \geqslant \frac{1}{2} \xi_{1} \ldots \xi_{p}^{\prime}$, sont de la forme

$$
x=\xi_{1} \ldots \xi_{p}\left(1-\xi_{p+1}\right)+\sum_{k \geqslant p+10} \alpha_{k} \xi_{1} \ldots \xi_{k}\left(1-\xi_{k+1}\right), \quad \alpha_{k} \in\{0,1\} .
$$

Nous envisageons de tels $x$, et nous notons

$$
\Psi(x)=\exp \frac{i \frac{\pi}{2} x}{\xi_{1} \ldots \xi_{p}\left(\xi_{p+1}-\zeta_{p+1}\right)},
$$

ce qui nous donne

$$
\begin{aligned}
& \Phi \circ g(x) \cdot \overline{\Psi(x)} \\
& =\exp i \frac{\pi}{2} \frac{\xi_{p+1}-\zeta_{p+1}+\sum \alpha_{k}\left[\zeta_{p+1} \ldots \zeta_{k}\left(1-\zeta_{k+1}\right)-\xi_{p+1} \ldots \xi_{k}\left(1-\xi_{k+1}\right)\right]}{\xi_{p+1}-\zeta_{p+1}}, \\
& |\Phi \circ g(x) \cdot \overline{\Psi(x)}-i| \leqslant \sum_{k \geqslant p+10} \frac{\pi}{2} \frac{\left|\zeta_{p+1} \ldots \zeta_{k}\left(1-\zeta_{k+1}\right)-\xi_{p+1} \ldots \xi_{k}\left(1-\xi_{k+1}\right)\right|}{\left|\xi_{p+1}-\zeta_{p+1}\right|} .
\end{aligned}
$$

La formule des accroissements finis pour les fonctions de plusieurs variables permet de transformer cette inégalité:

$$
\begin{aligned}
|\Phi \circ g(x) \cdot \overline{\Psi(x)}-i| & \leqslant \sum_{k \geqslant p+10} \sum_{j=p+1}^{k+1} \frac{\pi}{2}\left|\frac{\zeta_{j}-\xi_{j}}{\xi_{p+1}-\zeta_{p+1}}\right|\left(\frac{11}{20}\right)^{k-p-1} \\
& \leqslant \sum_{k \geqslant p+10} \frac{\pi}{2} \frac{k-p+1}{\left|\xi_{p+1}-\zeta_{p+1}\right|}\left(\frac{11}{20}\right)^{k-p+1} \sup _{j>p}\left|\zeta_{j}-\xi_{j}\right| \\
& \leqslant \frac{\pi}{2\left|\xi_{p+1}-\zeta_{p+1}\right|} \sup _{j>p}\left|\zeta_{j}-\xi_{j}\right| \int_{10}^{\infty} u\left(\frac{11}{20}\right)^{u} d u \\
& \leqslant \frac{\sup _{j>p}\left|\zeta_{j}-\xi_{j}\right|}{10\left|\xi_{p+1}-\zeta_{p+1}\right|} .
\end{aligned}
$$

Par ailleurs,

$$
\Phi \circ g\left(\xi_{1} \ldots \xi_{p+1}\right) \cdot \overline{\Psi\left(\xi_{1} \ldots \xi_{p+1}\right)}=\exp \left(-i \frac{\pi}{2}\right)=-i,
$$

et le même calcul que précédemment nous donne, pour tout élément du support de $\sigma_{p}$ satisfaisant $x \leqslant \frac{1}{2} \xi_{1} \ldots \xi_{p}$,

$$
|\Phi \circ g(x) \cdot \overline{\Psi(x)}+i| \leqslant \frac{\sup _{j>p}\left|\xi_{j}-\xi_{j}\right|}{10\left|\xi_{p+1}-\zeta_{p+1}\right|},
$$


ce qui nous permet de conclure, si l'on a pris soin de choisir un indice $p$ pour lequel

$$
2\left|\xi_{p+1}-\zeta_{p+1}\right| \geqslant \sup _{j>p}\left|\zeta_{j}-\xi_{j}\right|
$$

(ce qui est possible d'après le corollaire de la proposition 3),

$$
\left|\int_{E^{\prime}} \Phi \circ g(x) \cdot \overline{\Psi(x)} d \sigma_{p}\right| \geqslant \frac{(n-10) \log 2}{\xi_{1} \ldots \xi_{p+n}} .
$$

Si, en outre, $p$ est assez grand pour que

$$
\sup _{\alpha>p}\left(1-2 \xi_{q}\right) \leqslant n^{-2} 4^{-n}
$$

le lemme 2 est applicable, et permet de déduire de (12)

$$
\|\Phi \circ g\|_{\mathcal{A}\left(E^{\prime}\right)} \geqslant \frac{n-10}{11}
$$

ce qui nous donne le résultat puisque $n$ peut être choisi arbitrairement grand.

Ceci montre que, si $f$ définit un isomorphisme de $A\left(F^{\prime}\right)$ dans $A(E), g$ est linéaire dans un certain voisinage de l'origine: il existe $x_{0}$ tel que

$$
\text { si } x \leqslant x_{0}, g(x)=a\left(r_{1}, \ldots, r_{N-2}\right) x .
$$

Comme les $r_{j}$ sont des constantes, il semble que a est aussi une constante absolue, mais nous aurions pu en fait remplacer les $r_{j}$ par n'importe quel

$$
x_{j}=\sum_{k=1}^{k_{j}} \varepsilon_{k} r_{k}
$$

où les divers $\varepsilon_{k}$ sont choisis pour que la some des $x_{j}$ soit dans $E$. On peut alors envisager l'ensemble des constantes a ainsi obtenues.

Proposition II, 5. La fonction $a\left(x_{1}, \ldots, x_{N-2}\right)$ ne prend qu'un nombre fini de valeurs lorsque $x_{N-2}$ varie, $x_{1}, \ldots, x_{N-3}$ restant fixes.

Démonstration. Nous utiliserons une méthode très voisine de celle de [4]: nous posons tout d'abord, arec $i>N$,

$$
h\left(x_{i}, x\right)=\sum(-1)^{J} f\left(\sum_{j=1}^{N-3} \varepsilon_{j} x_{j}+\varepsilon_{i} x_{i}+x\right) .
$$

Cette fonction est (en particulier) définie sur

$$
\left\{x_{i_{1}}, x_{i_{2}}, \ldots, x_{i_{m}}\right\} \times\left\{x \in E ; x<\inf \left(r_{k_{1}}, \ldots, r_{k_{N-2}}, r_{k_{i_{1}}}, \ldots, r_{k_{i_{m}}}\right)\right\}
$$

et satisfait

$$
h\left(x_{i}, x\right)=h\left(x_{i}, 0\right)+a_{i} x, \quad a_{i}=a\left(x_{1}, \ldots, x_{N-3}, x_{i}\right)
$$

sur un ensemble de la forme

$$
E^{\prime \prime}=\left\{x_{i_{1}}, x_{i_{2}}, \ldots, x_{i_{m}}\right\} \times\left\{x \in E ; x \leqslant x_{0}\right\} .
$$

Comme, en outre,

$h$ est telle que

$$
\left\|e^{n i f}\right\|_{\mathcal{A}(E)} \leqslant C
$$

$$
\left\|e^{n i \hbar}\right\|_{A\left(E^{\prime}\right)} \leqslant C^{\left(2^{N}\right)} .
$$

En vue d'obtenir une inégalité contradictoire, nous définissons la mesure $\lambda_{0}$, unitaire équirépartie sur $\left\{x \in E ; x \leqslant x_{0}\right\}$, puis la mesure $\lambda_{i}$ portée par $x_{i} \times\left\{x \in E ; x \leqslant x_{0}\right\}$, et satisfaisant

$$
d \lambda_{i}\left(x_{i}, x\right)=\exp \left[-n i h\left(x_{i}, x\right)\right] d \lambda_{0}(x),
$$

ce qui nous permet d'assurer

$$
\sum_{i} \int_{\mathbb{E}^{\prime \prime}} e^{n i \hbar} d \lambda_{i}=m
$$

Comme en outre, le lemme 1 est applicable à $\lambda_{\theta}$ au lieu de $\lambda$, il existe $T$ tel que $|t| \geqslant T$ entraîne

$$
\left|\hat{\lambda}_{0}(t)\right| \leqslant \frac{1}{m}
$$

et nous pouvons alors, si les $a_{i}$ sont deux à deux distincts, déterminer $n$ tel que

$$
\nabla i, i^{\prime}, n\left|a_{i}-a_{i^{\prime}}\right| \geqslant 2 T,
$$

d'où nous déduisons, puisque $h\left(x_{i}, x\right)$ est linéaire de pente $a_{i}$

$$
\left\|\sum_{i} \lambda_{i}\right\|_{P M} \leqslant 2
$$

L'inégalité (14) devient alors

$$
\left\|e^{n i h}\right\|_{\Delta\left(\mathbb{E}^{\prime \prime}\right)} \geqslant \frac{m}{2}
$$

et, en comparant cette inégalité avec (13), on constate que les $a_{i}$ ne peurent prendre qu'un nombre fini de valeurs.

COROLLATRE. Il existe $x_{0}^{\prime}$ tel que, si pour $1 \leqslant j \leqslant N-2, x_{j} \leqslant x_{0}^{\prime}$,

$$
a\left(x_{1}, \ldots, x_{N-2}\right)=0 .
$$

Démonstration. En revenant à la définition de la fonction $g(x)$, on obtient, quel que soit $j$,

$a\left(x_{1}, \ldots, x_{j}+x_{j}^{\prime}, \ldots, x_{N-2}\right)=a\left(x_{1}, \ldots, x_{j}, \ldots, x_{N-2}\right)+a\left(x_{1}, \ldots, x_{j}^{\prime}, \ldots, x_{N-2}\right)$ 
et, comme la fonction $a$ ne peut prendre qu'un nombre fini de valeurs (on aurait pu, dans la proposition 5 , faire varier n'importe quel $x_{j}$ au lieu de $\left.x_{N-2}\right), a$ est nécessairement nulle lorsque tous les $x_{j}$ sont assez petits. Nous sommes alors en mesure de démontrer:

THÉORÈME II,1. Si $E$ est un ensemble parfait symétrique dont le rapport de dissection a pour limite $\frac{1}{2}$, si $f$ definit un isomorphisme de $A(F)$ dans $A(E)$, on peut écrire $[0,1]$ comme union finie de segments $I_{n}$ tels que la restriction de f à $E \cap I_{n}$ soit

$$
f(x)=a_{n} x+b_{n}, \quad a_{n} \in \boldsymbol{R}, b_{n} \in \boldsymbol{R} .
$$

Démonstration. A un ensemble $\left(x_{1}, \ldots, x_{N-2}\right)$ satisfaisant $x_{j} \leqslant x_{0}^{\prime}$, on peut associer $x_{0}$, dépendant des constantes $k_{0}, k_{1}$ et $k_{2}$ des propositions 2, 3 et 4 , et tel que, si $x \leqslant x_{0}, g(x)=0$. Or la constante $k_{0}$ a été introduite avant la définition de la fonction $g(x)$; elle est donc indépendante du choix de l'ensemble $\left(x_{1}, \ldots, x_{N-2}\right)$. De leur côté, $k_{1}$ et $k_{2}$ ne dépendent que de l'ensemble $E$ et de la norme de l'homomorphisme défini par $g$, qui est aussi majorée indépendamment de $\left(x_{1}, \ldots, x_{N-2}\right)$. Quitte à nous restreindre à l'ensemble

$$
\left\{\left(x_{1}, \ldots, x_{N-2}, x\right) ; x_{j} \leqslant \inf \left(x_{0}, x_{0}^{\prime}\right), x \leqslant \inf \left(x_{0}, x_{0}^{\prime}\right)\right\},
$$

nous obtenons alors $g(x)=0$, ce qui est une relation meilleure que celle donnée par le théorème $I, 1$ et nous pouvons répéter tout le raisonnement précédent à partir de cette nouvelle relation, et ainsi de suite... Il existe finallement un voisinage de zero sur lequel

$$
f(x+y)=f(x)+f(y)-f(0)
$$

et nous posons encore

$$
g(x)=f(x)-f(0)
$$

pour montrer que $g$ est une fonction linéaire, et donc que $f$ est une fonction affine. Il est toutefois utile de remarquer que, si $x_{0}$ a pu être minoré en fonction de la norme de l'homomorphisme défini par $g$, il n'en est pas de même pour $x_{0}^{\prime}$, et qu'il est done impossible de préciser la dimension du voisinage de zéro sur lequel $f$ est affine.

On peut cependant démontrer le même résultat au voisinage de tout point de $E$ de la forme

$$
x=\sum \varepsilon_{j} \xi_{1} \ldots \xi_{j-1}\left(1-\xi_{j}\right), \quad \sum \varepsilon_{j}<\infty, \varepsilon_{j} \epsilon\{0,1\},
$$

et on peut donc écrire $E$ comme union infinie d'ensembles $E_{n}=E \cap I_{n}$ tels que $x \in E_{n}$ entraîne

$$
f(x)=a_{n} x+b_{n} .
$$

Nous pouvons alors conclure en appliquant la méthode de [5]: une démonstration absolument identique à celle de la proposition 5 permet de montrer que les coefficients $a_{n}$ appartiennent nécessairement à un ensemble fini (si l'on ne veut pas refaire la démonstration dans ce cas, jl suffit d'appliquer le principe des soucoupes d'Y. Meyer [6] à la proposition 5).

Il reste donc à montrer que, s'il existe une suite infinie de segments $I_{n}$ tels que $x \in E \cap I_{n}$ entraîne

$$
f(x)=a x+b_{n},
$$

$b_{n}$ reste constant à partir d'un certain rang.

Soient $x_{k}$ et $x_{k}^{\prime}$ tels que

$$
\begin{aligned}
& x_{k}=\sum_{j \leqslant k} \varepsilon_{j} \xi_{1} \ldots \xi_{j-1}\left(1-\xi_{j}\right), \quad \varepsilon_{j} \in\{0,1\}, \\
& x_{k}^{\prime}=\sum_{j \leqslant k} \varepsilon_{j}^{\prime} \xi_{1} \ldots \xi_{j-1}\left(1-\xi_{j}\right), \quad \varepsilon_{j}^{\prime} \in\{0,1\}, \\
& f(x)=a x+b_{k} \quad \operatorname{sur}\left[x_{k}, x_{k}+\xi_{1} \ldots \xi_{k}\right] \cap E, \\
& f(x)=a x+b_{k}^{\prime} \quad \operatorname{sur}\left[x_{k}^{\prime}, x_{k}^{\prime}+\xi_{1} \ldots \xi_{k}\right] \cap \mathbb{D} .
\end{aligned}
$$

Si $v_{k}$ est la mesure unitaire équirépartie sur $\left[0, \xi_{1} \ldots \xi_{k}\right]$, nous posons

$$
d \omega_{k}(x)=e^{-N i f(x)} d v_{k}\left(x-x_{k}\right)+e^{-N i f(x)} d v_{k}\left(x-x_{k}^{\prime}\right),
$$

et la transformée de Fourier de $\omega_{k}$ est

$$
\left|\hat{\omega}_{k}(t)\right|=2\left|\cos \left[(t+N a) \frac{x_{k}-x_{k}^{\prime}}{2}+N \frac{b_{k}-b_{k}^{\prime}}{2}\right] \hat{y}_{k}(t+N a)\right| .
$$

Si $N$ est tel que, pour un entier $m,\left|N\left(b_{k}-b_{k}^{\prime}\right)-(2 m+1) \pi\right| \leqslant(2 q)^{-1}$, l'inégalité $\left|\omega_{l o}(t)\right| \leqslant 2 q^{-1}$ aura lieu lorsque

$$
|t+N a| \leqslant\left|\frac{1}{q\left(x_{k}-x_{k}^{\prime}\right)}\right| \quad \text { ou } \quad\left|\hat{\nu}_{k}(t+N a)\right| \leqslant \frac{1}{q} .
$$

On envisage alors une suite d'indices $k$, soit $k_{1}, \ldots, k_{q}$ tels que

$$
\frac{1}{q\left|x_{k_{j+1}}-x_{k_{j+1}}^{\prime}\right|} \geqslant t_{j}, \quad \sup _{|t|>t_{j}}\left|\hat{\nu}_{k_{j}}(t)\right| \leqslant \frac{1}{q} .
$$

Il n'existe alors pour chaque valeur de $t$ qu'un seul indice $j$ pour lequel l'inégalité $\left|\hat{\omega}_{k k_{j}}(t)\right| \leqslant 2 q^{-1}$ peut ne pas être réalisée, et on peut donc conclure, si l'on a pris soin de choisir une suite telle qu'il existe $N$ et $m_{j}$ satisfaisant

$$
\left|N\left(b_{k_{j}}-b_{k_{j}}^{\prime}\right)-\left(2 m_{j}+1\right) \cdot \pi\right| \leqslant \frac{1}{2 q}
$$


(ce qui est toujours possible pour une sous-suite assez lacunaire),

$$
\left\|\sum_{k} \omega_{k_{i}}\right\|_{P M} \leqslant 4
$$

Comme

$$
\sum_{k} \int_{t \in} e^{N i f(x)} d \omega_{k}=2 q
$$

le théorème provient de ce que $q$ doit restér fini.

\section{TROISIEME PARTIE}

Pour préciser la nature des homomorphismes d'algèbres de restriction dans le cas où le rapport de dissection de $E$ n'a plus pour limite $\frac{1}{2}$, nous aurons besoin de connaître non seulement $E$, mais aussi $f(E)$. Nous supposerons ici que $f(E)$ est lui-même contenu dans un ensemble parfait symétrique, et nous démontrerons un résultat qui nécessite deux définitions:

DÉfINITIon 1. Nous dirons que l'ensemble parfait symétrique

$$
E=\left\{x ; x=\sum_{j \geqslant 1} \varepsilon_{j} \xi_{1} \ldots \xi_{j-1}\left(1-\xi_{j}\right), \varepsilon_{j} \in\{0,1\}\right\}
$$

satisfait la condition $M$ si, quels que soient $\eta$ et $P$ positifs fixés, on peut trouver $T$ tel que, pour tout sous-ensemble $A$ de $N$ satisfaisant

$$
\text { card } A \leqslant P \text {, }
$$

l'ensemble parfait symétrique

$$
E_{A}=\left\{x ; x=\sum_{j \notin A} \varepsilon_{j} \xi_{1} \ldots \xi_{j-1}\left(1-\xi_{j}\right), \varepsilon_{j} \in\{0,1\}\right\}
$$

porte une mesure positive unitaire $\mu_{A}$ telle que

$$
\sup _{\mid t \geqslant T}\left|\hat{\mu}_{\mathcal{A}}(t)\right| \leqslant \eta \text {. }
$$

Défunition 2. Nous dirons que l'ensemble parfait symétrique

$$
F=\left\{z ; z=\sum_{k \geqslant 1} \varepsilon_{k} \zeta_{1} \ldots \zeta_{k-1}\left(1-\zeta_{k}\right), \varepsilon_{k} \in\{0,1\}^{*}\right\}
$$

satisfait la condition $U$ s'il existe une constante $\theta$ et une suite de fonctions $\left\{\Phi_{k}\right\}$ de norme bornée dans $A(F)$ telle que, si

$$
\begin{aligned}
& z_{1}=\sum_{i \neq k} \varepsilon_{i} \zeta_{1} \ldots \zeta_{i-1}\left(1-\zeta_{i}\right), \quad \varepsilon_{i} \in\{0,1\}, \\
& z_{2}=z_{1}+\zeta_{1} \ldots \zeta_{k-1}\left(1-\zeta_{k}\right)
\end{aligned}
$$

alors

$$
\text { Rée } \Phi_{k}\left(z_{1}\right) \geqslant 1-\theta, \quad \text { Ré } \Phi_{k}\left(z_{2}\right) \leqslant 1-2 \theta \text {. }
$$

THÉORغ̀ME III, 1 . Si $E$ satisfait la condition $M$, si $F$ satisfait la contit.on $U$, les homomorphismes de $A(F)$ dans $A(E)$ sont donnés par les applications $f$ de $E$ dans $F$ telles que $f(E)$ ne contient $q u$ 'un nombre fini d'éléments.

Démonstration. Les homomorphismes de $A(E)$ dans $A(E)$ sont donnés par des applications $f$ de $E$ dans $F$ satisfaisant le théorème I,2, que nous pouvons préciser en écrivant que $f(E) \subset F$ :

$$
f(x)=\sum_{m<N} f_{m}(x)
$$

et, si l'on note $r_{j}=\xi_{1} \ldots \xi_{j-1}\left(1-\xi_{j}\right), f_{m}(x)$ est telle que

$$
\begin{gathered}
f_{m}\left(\sum r_{j}\right)=\sum f_{m}\left(r_{j_{1}}+\ldots+r_{j_{m}}\right), \\
f_{m}\left(r_{j_{1}}+\ldots+r_{j_{m}}\right)=\sum_{k \geqslant 1} \alpha_{m, k} \zeta_{1} \ldots \zeta_{k-1}\left(1-\zeta_{k}\right), \quad\left|\alpha_{m, k}\right| \leqslant m ! .
\end{gathered}
$$

Nous utiliserons ces résultats en notant

$$
\begin{aligned}
& x=\sum_{j \geqslant 1} \varepsilon_{j} \xi_{1} \ldots \xi_{j-1}\left(1-\xi_{j}\right), \quad \varepsilon_{j} \epsilon\{0,1\} \\
& f(x)=\sum_{k \geqslant 1} \varepsilon_{k}^{\prime} \zeta_{1} \ldots \zeta_{k-1}\left(1-\zeta_{k}\right), \quad \varepsilon_{k}^{\prime} \in\{0,1\}
\end{aligned}
$$

et en étudiant tout d'abord la correspondance ainsi définie entre une suite $\left\{\varepsilon_{j}\right\}$ et une suite $\left\{\varepsilon_{k}^{\prime}\right\}$. Pour 7 fixé, la valeur de $\varepsilon_{k}^{\prime}$ dépend d'un nombre fini de valeurs de la suite $\left\{\varepsilon_{j}\right\}$, soit au plus $\omega(N):$ si cela n'était pas vrai, on pourrait en effet, puisque le nombre de tous les $a_{m, k}$ (correspondant à toutes les valeurs de $m$ ) est borné en fonction de $N$, trouver un ensemble $J$ de $N$ indices $j$, tel que les valeurs des $\alpha_{m, k}$, non toutes nulles, restent constantes lorsque, $k$ et $m$ étant fixés, les indices $j_{1}, \ldots, j_{m}$ varient dans $J$. Nous envisageons alors une suite $\left\{\varepsilon_{j}\right\}$ telle que

$$
\begin{gathered}
\text { si } j \notin J, \quad \varepsilon_{j}=0, \\
\operatorname{Card}\left\{j ; j \in J, \varepsilon_{j} \neq 0\right\}=M,
\end{gathered}
$$

et nous pouvons calculer la valeur du $\varepsilon_{k}^{\prime}$ correspondant:

$$
\varepsilon_{l i}^{\prime}=\left(\begin{array}{c}
M \\
N-1
\end{array}\right) \alpha_{N-1, k}+\left(\begin{array}{c}
M \\
N-2
\end{array}\right) \alpha_{N-2, k}+\ldots+\left(\begin{array}{c}
M \\
0
\end{array}\right) a_{0, k} .
$$

Les coefficients $\alpha_{m, k}$ sont done solutions d'un système de $N+1$ équations indépendantes à $N$ inconnues. Ceci n'est possible que si $\alpha_{m, k}=0$ quel que soit $m$, et nous obtenons donc une contradiction, ce qui prouve que $\varepsilon_{k}^{\prime}$ dépend de moins de $\omega(N)$ valeurs de la suite $\left\{\varepsilon_{j}\right\}$. 
Comme nous n'arons pas supposé que $f$ est surjective, il peut par ailleurs exister des indices $k$ tels que $\varepsilon_{k}^{\prime}$ soit indépendant de $x$, et donc de la suite $\left\{\varepsilon_{j}\right\}$. Il peut en outre exister un entier $q_{1}$ tel que la condition

$$
\varepsilon_{j}=0 \quad \text { si } j \geqslant q_{1}
$$

ne permet, pour aucun des indices $k$ tels que $\varepsilon_{k}^{\prime}$ dépend de $x$, de préciser la valeur de $\varepsilon_{k}^{\prime}$. Nous étudions alors la restriction de $f$ à $\left\{x \in E ; x \leqslant \xi_{1} \ldots\right.$ $\left.\ldots \xi_{q_{1}}\right\}$, et nous répétons le raisonnement précédent: il peut exister des indices $k$ tels que $\varepsilon_{k}^{\prime}$ soit indépendant de l'élément $x$ de cet ensemble (et alors, la construction de $q_{1}$ montre que $\varepsilon_{k}^{\prime}$ est indépendant de $x \in E$ ). II peut aussi exister un entier $q_{2}$ tel que la condition

$$
\varepsilon_{j}=0 \text { si } j<q_{1} \text { ou si } j \geqslant q_{2}
$$

ne permet, pour aucun des indices $k$ tels que $\varepsilon_{k}^{\prime}$ dépend de $x$, de préciser la valeur de $\varepsilon_{k}^{\prime} \ldots$ Nous construisons ainsi la suite $\left\{q_{1}, q_{2}, \ldots\right\}$ et le résultat précédent montre que cette suite contient au plus $\omega(N)$ éléments.

Soit $q_{n}$, le dernier terme de la suite; nous étudions alors la restriction de $f$ à $\left\{x \in t ; x \leqslant \xi_{1} \ldots \xi_{q_{n}}\right\}$, ou, ce qui revient au même, nous supposons qu'il est impossible de déterminer $q_{1}$. Nous remarquons en effet que le théorème I, 2 implique que, si $f(E)$ contient une infinité d'éléments, il en est de même de $f\left(\left\{x \in E ; x \leqslant \xi_{1} \ldots \xi_{q_{n}}\right\}\right)$.

Ceci revient à dire que l'on peut déterminer $j_{0}$ arbitrairement grand et $k_{0}$ tels que

$$
\begin{gathered}
\varepsilon_{k_{0}}^{\prime} \text { dépend effectivement de } x, \\
\nabla j \leqslant j_{0}, \quad \varepsilon_{k_{0}}^{\prime} \text { ne dépend pas de } \varepsilon_{j} .
\end{gathered}
$$

Nous notons alors $A=\left\{j ; \varepsilon_{k_{0}}^{\prime}\right.$ dépend de $\left.\varepsilon_{j}\right\}$, et nous savons déjà que $A$ contient au plus $\omega(N)$ éléments. Nous pouvons done utiliser le fait que $E$ satisfait la condition $M$ pour assurer que

$$
E_{A}=\left\{x ; x=\sum_{j \neq A} \varepsilon_{j} \xi_{1} \ldots \xi_{j-1}\left(1-\xi_{j}\right), \varepsilon_{j} \in\{0,1\}\right\}
$$

porte une mesure positive $\mu_{A}$ telle que

$$
\sup _{|t| \geqslant T}\left|\hat{\mu}_{A}(t)\right| \leqslant \eta \text {. }
$$

En fait, $N$ et $\eta$ étant fixés, $T$ est donné par la condition $M$, et nous choisissons $j_{0}$ de façon que

$$
\xi_{1} \ldots \xi_{j_{0}} \leqslant 2 \frac{\eta}{T}
$$

puis nous définissons la mesure

$$
v_{A}(x)=\mu_{A}(x)-\mu_{A}\left(x-x_{A}\right)
$$

où

$$
x_{A}=\sum_{j \in A} \varepsilon_{j} \xi_{1} \ldots \xi_{j-1}\left(1-\xi_{j}\right), \quad \varepsilon_{j} \in\{0,1\}
$$

est choisi de telle sorte que l'on puisse par exemple affirmer que, quel que soit $x \in E_{A}$,

$$
\begin{gathered}
f(x)=\sum_{k \geqslant 1} \varepsilon_{k} \zeta_{1} \ldots \zeta_{k-1}\left(1-\zeta_{k}\right), \quad \varepsilon_{k} \in\{0,1\}, \varepsilon_{k_{0}}=0 \\
f\left(x+x_{A}\right)=\sum_{k \geqslant 1} \varepsilon_{k}^{\prime} \zeta_{1} \ldots \zeta_{k-1}\left(1-\zeta_{k}\right), \quad \varepsilon_{k}^{\prime} \in\{0,1\}, \varepsilon_{k_{0}}^{\prime}=1
\end{gathered}
$$

Comme

$$
\left|\hat{v}_{A}(t)\right| \leqslant \xi_{1} \ldots \xi_{j_{0}}\left|\hat{\mu}_{A}(t)\right|
$$

et

$$
\left|\hat{\nu}_{A}(t)\right| \leqslant 2\left|\hat{\mu}_{A}(t)\right|,
$$

on peut dans tous les cas assurer

$$
\left|\hat{v}_{A}(t)\right| \leqslant 2 \eta .
$$

Par ailleurs,

$$
\operatorname{Ré} \int_{E} \Phi_{k} \circ f d v_{A} \geqslant \theta
$$

et nous obtenons donc, en comparant les deux dernières inégalités,

$$
\left\|\Phi_{k} \circ f\right\|_{\mathcal{A}(E)} \geqslant \frac{\theta}{2 \eta}
$$

ce qui montre que $f$ ne définit pas un homomorphisme de $A(F)$ dans $A(E)$, puisque $\theta$ est fixe, et que $\eta$ peut être choisi arbitrairement petit.

Comme il est difficile de montrer qu'un ensemble satisfait la condition $U$, nous énonçons maintenant une nouvelle condition, et nous démontrons alors un théorème plus faible que le théorème 1:

DÉfinimion 3. Nous dirons que l'ensemble parfait symétrique

$$
F=\left\{z ; z=\sum_{k \geqslant 1} \varepsilon_{k} \zeta_{1} \ldots \zeta_{k-1}\left(1-\zeta_{k}\right), \varepsilon_{k} \in\{0,1\}\right\}
$$

satisfait la condition $\sigma^{\prime}$ s'il existe deux constantes $K$ et $\theta$, et une suite de fonctions $\left\{\Phi_{k}\right\}$ de norme bornée dans $A\left(F^{\prime}\right)$ telle que, si

$$
\begin{aligned}
& z_{1}=\sum_{\left|i-k_{k}\right|>K} \varepsilon_{i} \zeta_{1} \ldots \zeta_{i-1}\left(1-\zeta_{i}\right), \quad \varepsilon_{i} \epsilon\{0,1\}, \\
& z_{2}=z_{1}+\zeta_{1} \ldots \zeta_{k-1}\left(1-\zeta_{k}\right)
\end{aligned}
$$


alors

$$
\text { Ré } \Phi_{l c}\left(z_{1}\right) \geqslant 1-\theta, \quad \text { Ré } \Phi_{k}\left(z_{2}\right) \leqslant 1-2 \theta .
$$

THÉoß亡̀me III,2. Si $E$ satisfait la condition $M$, si $F$ satisfait la condition $U^{\prime}, A(E)$ et $A(F)$ ne sont pas isomorphes.

Démonstration. Nous suivons la démonstration du théorème 1 , et nous définissons ainsi la suite $\left\{q_{1}, q_{2}, \ldots\right\}$ : la condition

$$
\varepsilon_{j}=0 \quad \text { si } j<q_{i} \text { ou si } j \geqslant q_{i+1}
$$

ne permet, pour aucun des indices $k$, de préciser la valeur de tous les $\varepsilon_{m}^{\prime}$ correspondant à des indices tels que $|m-k| \leqslant K$.

Si nous notons encore $q_{n} \leqslant(2 K+1) \omega(N)$, le dernier terme de cette suite, et $E_{n}=\left\{x \in E ; x \leqslant \xi_{1} \ldots \xi_{q_{n}}\right\}, f$ définit une bijection de $E_{n}$ sur $f\left(E_{n}\right)$, et la continuité de $f^{-1}$ entraîne l'existence d'un indice $p_{n}$ tel que $F \cap\left[0, \zeta_{1} \ldots \zeta_{p_{n}}\right]$ (ou l'un des translatés de cet ensemble) soit contenu dans $f\left(E_{n}\right)$. On détermine alors $j_{0}$ arbitrairement grand, et $k_{0}$ supérieur à $p_{n}+K$, tels que

$$
\nabla j \leqslant j_{0}, \nabla k,\left|k-k_{0}\right| \leqslant K, \varepsilon_{k}^{\prime} \text { ne dépend pas de } \varepsilon_{j},
$$

et l'on note $A=\left\{j\right.$; $1 \mathrm{H} k,\left|k-k_{0}\right| \leqslant K, \varepsilon_{k}^{\prime}$ dépend de $\left.\varepsilon_{j}\right\}$.

On recopie alors la fin de la démonstration du théorème 1 , en remarquant que l'on peut choisir $x_{A}$ tel que, si $x \in E_{A}$,

$$
\begin{gathered}
f(x)=\sum_{\left|k-k_{0}\right|>K} \varepsilon_{k} \zeta_{1} \ldots \zeta_{k-1}\left(1-\zeta_{k}\right), \quad \varepsilon_{k} \in\{0,1\}, \\
f\left(x+x_{A}\right)=\zeta_{1} \ldots \zeta_{k_{0}-1}\left(1-\zeta_{k_{0}}\right)+\sum_{\left|k-k_{0}\right|>K} \varepsilon_{k}^{\prime} \zeta_{1} \ldots \zeta_{k-1}\left(1-\zeta_{k}\right), \quad \varepsilon_{k}^{\prime} \in\{0,1\} .
\end{gathered}
$$

Nous allons maintenant donner des applications des théorèmes 1 et 2 , en mettant en évidence des ensembles satisfaisant soit la condition $M$, soit la condition $U$, soit la condition $U^{\prime}$ :

THÉORÈME III, 3. Si $\xi_{j}$ est constant et égal à un nombre $\xi$ dont l'inverse n'est pas un nombre de Pisot, l'ensemble parfait symétrique $E$, à rapport de dissection constant $\xi$, satisfait la condition $M$.

Démonstration. Pour $P$ positif fixé, il existe $n>P$ tel que $\xi^{-n}$ n'est pas un nombre de Pisot. Les mesures unitaires équiréparties sur les ensembles

$$
E_{k}=\left\{x ; x=\xi^{k}(1-\xi) \sum_{j \geqslant 1} \varepsilon_{j} \xi^{j n}, \varepsilon_{j} \in\{0,1\}\right\}
$$

ont une transformée de Fourier qui tend vers 0 à l'infini, et comme il existe $n$ ensembles $E_{k}$, on obtient le résultat, si l'on a pris soin de choisir $P \geqslant \operatorname{Card} A$, de façon à pouvoir assurer qu'il existe un indice $k_{\mathrm{u}}$ tel que
$E_{k} \subset E_{A}:$ on peut trouver $T$ ne dépendant que de $\eta$, et choisir pour $\mu_{A}$ la mesure unitaire équirépartie sur $E_{k} \subset E_{A}$.

THÉORł̀me III, 4. Si $\zeta_{j}$ est constant et égal à l'inverse d'un nombre entier $p, p \geqslant 3$, l'ensemble parfait symétrique $F$, à rapport de dissection constant $1 / p$ satisfait la condition $U$.

Démonstration. Nous posons, pour $z \in F$

$$
\Phi_{k}(z)=\Phi\left(\frac{p^{k-1}}{p-1} z\right)
$$

et nous déterminons $\Phi$ pour que $\Phi_{k}$ sépare les points de $F$ selon que $\varepsilon_{k}$ vaut 0 ou 1: il suffit de choisir pour $\Phi$, la fonction continue, de période 1 , telle que

$$
\begin{array}{ll}
\Phi(z)=0 & \text { sur }\left[\frac{1}{p}, \frac{1}{p-1}\right], \\
\Phi(z)=1 & \operatorname{sur}\left[0, \frac{1}{p(p-1)}\right],
\end{array}
$$

$\Phi(z)$ linéaire dans les intervalles.

Nous pouvons alors affirmer que $\Phi$ est la transformée de Fourier d'une mesure bornée portée par $\boldsymbol{R}$, ce qui entraîne que $\left\|\Phi_{l \|}\right\|_{A\left(F^{\prime}\right)}$ reste bornée.

THúforìme III, 5. Si $\zeta_{j}$ est constant et égal à l'inverse $\zeta$ d'un nombre de Pisot, l'ensemble parfait symétrique $F$, à rapport de dissection constant $\zeta$, satisfait la condition $U^{\prime}$.

Démonstration. Nous posons encore

$$
\Phi_{k}(z)=\Phi\left(\frac{\zeta^{2-k}}{1-\zeta} z\right)
$$

où $\Phi$ est la fonction continue, de période 1 , relle que

$$
\begin{aligned}
& \Phi(z)=0 \text { sur }\left[\frac{2}{3} \zeta+\frac{1}{3} \frac{\zeta^{2}}{1-\zeta}, \frac{2}{3} \frac{\zeta}{1-\zeta}+\frac{1}{3}\right], \\
& \Phi(z)=1 \text { sur }\left[-\frac{1}{3}+\frac{1}{3} \frac{\zeta}{1-\zeta}, \frac{1}{3} \zeta+\frac{2}{3} \frac{\zeta^{2}}{1-\zeta}\right],
\end{aligned}
$$

$\Phi(z)$ linéaire dans les intervalles.

$\left\|\Phi_{k}\right\|_{A(F)}$ reste bornée pour la même raison qu'au théorème 4 , et on obtient le résultat en choisissant $K$ assez grand, car la distance de $\zeta^{-k}$ à l'entier le plus proche tend exponentiellement vers 0 . 
THÉORÈME III, 6 . Si $\zeta_{j}$ est le terme général d'une série convergente, l'ensemble parfait symétrique $F$, défini par la suite de rapports de dissection $\left\{\zeta_{j}\right\}$ satisfait $l_{a}$ condition $U$.

Démonstration. Nous posons cette fois

$$
\Phi_{k}(z)=\exp \left(2 i \pi Z_{k} z\right) \quad \operatorname{sur}\left[0, \zeta_{1} \ldots \zeta_{j_{1}}\right]
$$

et nous allons préciser les valeurs de $Z_{k}$ et de $j_{1}$

Nous poserons alors, si $z=z_{0}+z_{1}$, avec

$$
z_{0}=\sum_{j \leqslant j_{1}} \varepsilon_{j} \zeta_{1} \ldots \zeta_{j-1}\left(1-\zeta_{j}\right), \quad z_{1} \leqslant \zeta_{1} \ldots \zeta_{j_{1}},
$$

$\Phi_{k}(z)=\Phi_{k_{k}}\left(z_{1}\right)$, ce qui nous permet d'affirmer, puisque $j_{1}$ est une constante absolue, que $\left\|\Phi_{z_{z}}\right\|_{\mathcal{A}\left(F^{\prime}\right)}$ reste bornée.

Nous définissons la suite $\left\{u_{1}, u_{2}, \ldots, u_{k}\right\}$ par

$$
\begin{gathered}
u_{1} \zeta_{1} \ldots \zeta_{k-1}\left(1-\zeta_{k}\right)=\frac{1}{2}, \\
\left|\left(u_{2}-u_{1}\right) \zeta_{1} \ldots \zeta_{k-2}\left(1-\zeta_{k-1}\right)\right| \leqslant \frac{1}{2}, \quad u_{2} \zeta_{1} \ldots \zeta_{k-2}\left(1-\zeta_{k-1}\right) \in \mathbf{N}, \\
\ldots \ldots \ldots \ldots \ldots \ldots \ldots \ldots \ldots \ldots \\
\left|\left(u_{j}-u_{j-1}\right) \zeta_{1} \ldots \zeta_{k-j}\left(1-\zeta_{k-j+1}\right)\right| \leqslant \frac{1}{2}, \quad u_{j} \zeta_{1} \ldots \zeta_{k-j}\left(1-\zeta_{k-j+1}\right) \in \boldsymbol{N}, \\
\ldots \ldots \ldots \ldots \ldots \ldots
\end{gathered}
$$

et nous posons $Z_{k}=u_{k}$, d'où nous déduisons

$$
\begin{aligned}
\left|Z_{k} \zeta_{1} \ldots \zeta_{k-1}\left(1-\zeta_{k}\right)-\frac{1}{2}\right| & =\left(u_{k}-u_{1}\right) \zeta_{1} \ldots \zeta_{k-1}\left(1-\zeta_{k}\right) \\
& \leqslant \sum_{j=1}^{k-1}\left|u_{j}-u_{j-1}\right| \zeta_{1} \ldots \zeta_{k-1}\left(1-\zeta_{k}\right) \\
& \leqslant 2 \zeta_{k-1} .
\end{aligned}
$$

Nous obtenons de la même façon

$$
\left|Z_{k}-u_{j}\right| \zeta_{1} \ldots \zeta_{k-j}\left(1-\zeta_{k-j+1}\right) \leqslant 2 \zeta_{k-j}
$$

et, si nous avons choisi $j_{1}$ tel que

$$
\sum_{j \geqslant j_{1}} 2 \zeta_{j} \leqslant \frac{1}{6}
$$

nous constatons que les fonctions $\Phi_{k}$ ainsi déterminées permettent d'affirmer que $F$ satisfait la condition $\quad J$, et nous obtenons alors $\theta=\frac{1}{3}$.

Remarque. Les applications de ce dernier résultat découlent presque toutes du théorème de Y. Meyer [2]; toutefois, en dehors du fait qu'il s'agit ici d'un résultat ,sûr", l'intérêt du théorème 6 est de montrer le rapport qui existe entre la condition $U$ et la notion d'unicité. Le théorème
6 n'est d'ailleurs certainement pas le meilleur résultat possible; il donne cependant une bonne idée de la nature de la condition $U$.

Nous noterons pour terminer, que la méthode utilisée ici permet aussi, dans certains cas où $E$ et $F$ sont deux ensembles de multiplicité, de montrer que $A(E)$ et $A(F)$ ne sont pas isomorphes. Nous ne ferons la démonstration que dans un cas particulier:

THÉ́Ṙ̀Me III,7. Si $E$ est un ensemble parfait symétrique défini par un rapport de dissection constant $\xi$, si $F$ est un ensemble parfait symétrique défini par une suite de rapports de dissection $\zeta_{k}$ tendant vers zéro, si $E$ et $F$ portent tous deux une mesure dont la transformée de Fourier tend vers zéro à l'infini, $A(E)$ et $A\left(F^{\prime}\right)$ ne sont pas isomorphes.

Démonstration. Si $\mu$ est une mesure telle que $\lim _{t} \hat{\mu}(t)=0$, alors

$$
\lim _{|t| \rightarrow \infty}|\mu|^{\wedge}(t)=0 .
$$

Si $f$ définit un isomorphisme de $A(F)$ dans $A(E)$, le théorème I,2 est applicable à $f$ comme à $f^{-1}$, et nous voyons, en suivant la démonstration du théorème III, 1 , que chaque $\varepsilon_{k}^{\prime}$ dépend encore de moins de $\omega(N)$ valeurs de $\varepsilon_{j}$, et que chaque $\varepsilon_{j}$ influe maintenant sur moins de $\omega(N)$ valeurs de $\varepsilon_{k}^{\prime}$. Comme $E$ satisfait la condition $M$ (théorème III,3), nous associons à $\eta$ et $P=\omega(N)$ un entier $m$ tel que, dans la définition 1, on puisse choisir $T=\xi^{-m}$, et nous déterminons $k_{1}$ tel que

$$
\sup _{k \geqslant k_{1}} \zeta_{k} \leqslant \frac{1}{4 m \omega(N)}
$$

puis $j_{1}$ tel que, si $E_{1}=E \cap\left[0, \zeta^{j_{1}}\right], f\left(E_{1}\right)$ est contenu dans un translaté de $F \cap\left[0, \zeta_{1} \ldots \zeta_{k_{2}}\right]$; nous notons alors $F_{1}$, le plus petit ensemble parfait symétrique tel que $f\left(E_{1}\right) \subset F_{1}$ (pour simplifier les notations, nous supposons dans la suite que $\left.F_{1}=E \cap\left[0, \zeta_{1} \ldots \zeta_{k_{1}}\right]\right)$.

Nous pouvons alors déterminer $j_{2} \geqslant j_{1}+m$, et $k_{2} \leqslant k_{1}+m \omega(N)$, tels que

$$
\nabla j \leqslant j_{2}, \quad \varepsilon_{k_{2}}^{\prime} \text { ne dépend pas de } \varepsilon_{j}
$$

et noter $A=\left\{j ; \varepsilon_{k_{2}}^{\prime}\right.$ dépend de $\left.\varepsilon_{j}\right\}$. En utilisant l'inégalité

$$
\sum_{k_{2} \geqslant k \geqslant k_{1}} \zeta_{k} \leqslant \frac{1}{4}
$$

la démonstration du théorème III, 6 nous permet de déterminer une fonction $\Phi$, de norme 1 dans $A\left(F^{\prime}\right)$, telle que, si

$$
z=\sum_{k \geqslant k_{1}} \varepsilon_{k}^{\prime} \zeta_{1} \ldots \zeta_{k-1}\left(1-\zeta_{k}\right), \quad\left|\operatorname{Ré} \Phi(z)-\varepsilon_{k_{2}}^{\prime}\right| \leqslant \frac{1}{4} .
$$


On peut alors reproduire la fin de la démonstration du théorème III,1, pour conclure

$$
\|\Phi \circ f\|_{A(E)} \geqslant \frac{1}{4 \eta},
$$

ce qui achève la démonstration puisque $\eta$ est arbitraire.

\section{Bibliographie}

[1] J.-P. Kahane, Séries de Fourier absolument convergentes, 1970

[2] Y. Meyer, Algebres de restriction non isomorphes, Ann. Inst. Fourier 19 (1969), p. $117-124$.

[3] A. B eurling et H. Helson, Fourier-Stieltjes transforms with bounded powers, Math. Scand. I (1953), p. 120-126.

[4] N. Leblanc, Sur les isomorphismes dérivables des algèbres de restriction, C. R. Acad. Sci. Paris 270 (1970), A520-A522.

[5] - Les enḋomorphismes d'algèbre à poids, Bull. Soc. Math. France 99 (1971), p. $387-396$.

[6]. Y. Meyer, Isomorphismes entre certaines algèbres de restriction, C. R. Acad. Sci. Paris 265 (1967), A18-A20.

CENTRE SCITNTIFIQUE ET POLTTECHNIQUT

SAINT-DENIS, FRANCT

\section{On weak compactness*}

by

MANUEL VALDIVIA (Valencia)

Abstract. In [1] E. E. Floyd and V. L. Klee have proved that a bounded closed convex subset $O$ of a normed linear space fails to be weakly compact if and only if there is a decreasing sequence of closed linear manifolds whose intersection is empty and each of which intersects $O$. We obtain here some properties f other results of V. Pták and R. C. James (see [5] and [2]).

We use here vector spaces over the field $K$ of real or complex numbers. When we use the word "space" it means "locally convex separated vector space". For every space $E$ we denote, as usual, by $E^{\prime}$ and $E^{*}$ its topological and algebraical dual, respectively.

LeMara. Given a space $E$ let $M$ be a dense subset of $E^{\prime}\left[\sigma\left(E^{\prime}, E\right)\right]$ with cardinal number $\alpha$. Let $z$ be a point of $\left(E^{\prime}\right)^{*}$. If $G$ is the linear hull of $E \cup\{z\}$ there exists in $E^{\prime}\left[\sigma\left(E^{\prime}, G\right)\right]$ a dense subset with cardinal number non-larger than $\alpha$.

Pro of. Suppose that $z$ is not in $E$. Let $L$ be the subspace of $E^{\prime}\left[\sigma\left(E^{\prime}, E\right)\right]$ generated by $M$. If the restriction of $z$ to $L$ is not continuous we put $L=P$. If the restriction of $z$ to $L$ is continuous let $y$ be a point of $E$ such that $\left\langle y, x^{\prime}\right\rangle=\left\langle z, x^{\prime}\right\rangle$, for all $x^{\prime}$ of $L$. Then there exists a $z^{\prime} \in E^{\prime}$ such that $\left\langle y, z^{\prime}\right\rangle \neq\left\langle z, z^{\prime}\right\rangle$ and therefore, if $P$ is the subspace of $E^{\prime}\left[\sigma\left(E^{\prime}, E\right)\right]$ generated by $L \cup\left\{z^{\prime}\right\}$, we have that the restriction of $z$ to $P$ is not continuous. The subspace $P \cap z^{-1}(0)$ is dense in $P$ and also in $E^{\prime}\left[\sigma\left(E^{\prime}, E\right)\right]$. We take $x_{0}^{\prime} \in P, x_{0}^{\prime} \notin z^{-1}(0)$. Given any $x^{\prime}$ of $E^{\prime}$ we can write

$$
x^{\prime}=y^{\prime}+\lambda x_{0}^{\prime}, \quad y^{\prime} \in z^{-1}(0), \lambda \in K \text {. }
$$

Let $\left\{x_{d}^{\prime}: d \in D\right\}$ be a net in $P \cap z^{-1}(0)$ which converges to $y^{\prime}$ for the topology $\sigma\left(E^{\prime}, E\right)$. We shall show that the net $\left\{x_{d}^{\prime}+\lambda x_{0}^{\prime}: d \in D\right\}$ converges to $x^{\prime}$ for the topology $\sigma\left(E^{\prime}, G^{\prime}\right)$. Indeed, if $x \in E$ we have that

* Supported in part by the "Patronato para el Fomento de la Investigación en la Universidad". 